\title{
AORTIC VALVE-PRESERVING PROCEDURE FOR ENLARGEMENT OF THE LEFT VENTRICULAR OUTFLOW TRACT AND MITRAL ANULUS
}

Richard A. Jonas, MD, John F. Keane, MD, and James E. Lock, MD, Boston, Mass.

From the Departments of Cardiac Surgery and Cardiology, Children's Hospital, and Department of Pediatrics, Harvard Medical School, Boston, Mass.

Received for publication Feb. 11, 1997; accepted for publication Dec. 4, 1997.

Address for reprints: Richard A. Jonas, MD, Children's Hospital, Department of Cardiac Surgery, 300 Longwood Ave., Boston, MA 02115.

J Thorac Cardiovasc Surg 1998;115:1219-22

Copyright (C) 1998 by Mosby, Inc.

$0022-5223 / 98 \$ 5.00+0 \quad \mathbf{1 2 / 5 4 / 8 8 0 1 4}$
Tunnel subaortic stenosis is often a component of Shone's syndrome and therefore may be associated with mitral stenosis and hypoplasia of the mitral anulus. ${ }^{1}$ It is also often, although not always, associated with valvular aortic stenosis. Patch enlargement of the ventricular septum, which forms the anterior wall of the left ventricular outflow tract, with preservation of the aortic anulus and valve ("modified Konno procedure"), is a useful procedure for management of tunnel subaortic stenosis when the aortic anulus and mitral anulus are of adequate size. However, when the mitral anulus is hypoplastic and must be enlarged to accommodate an adequate-sized prosthe- 


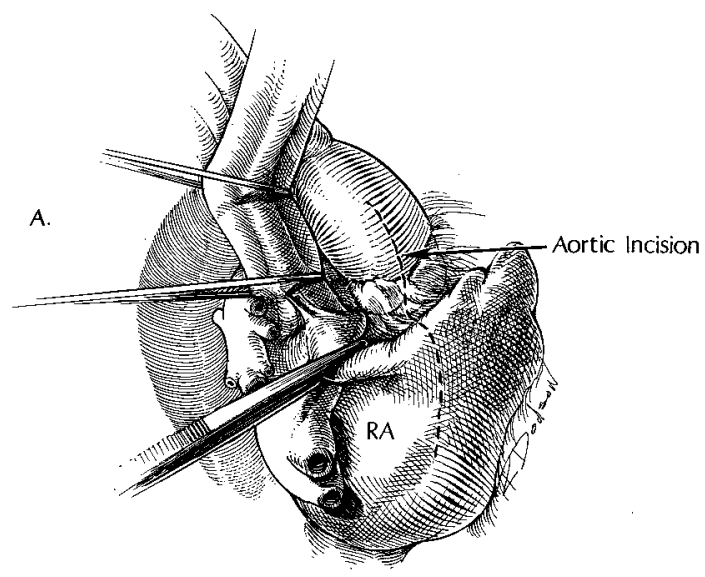

B.
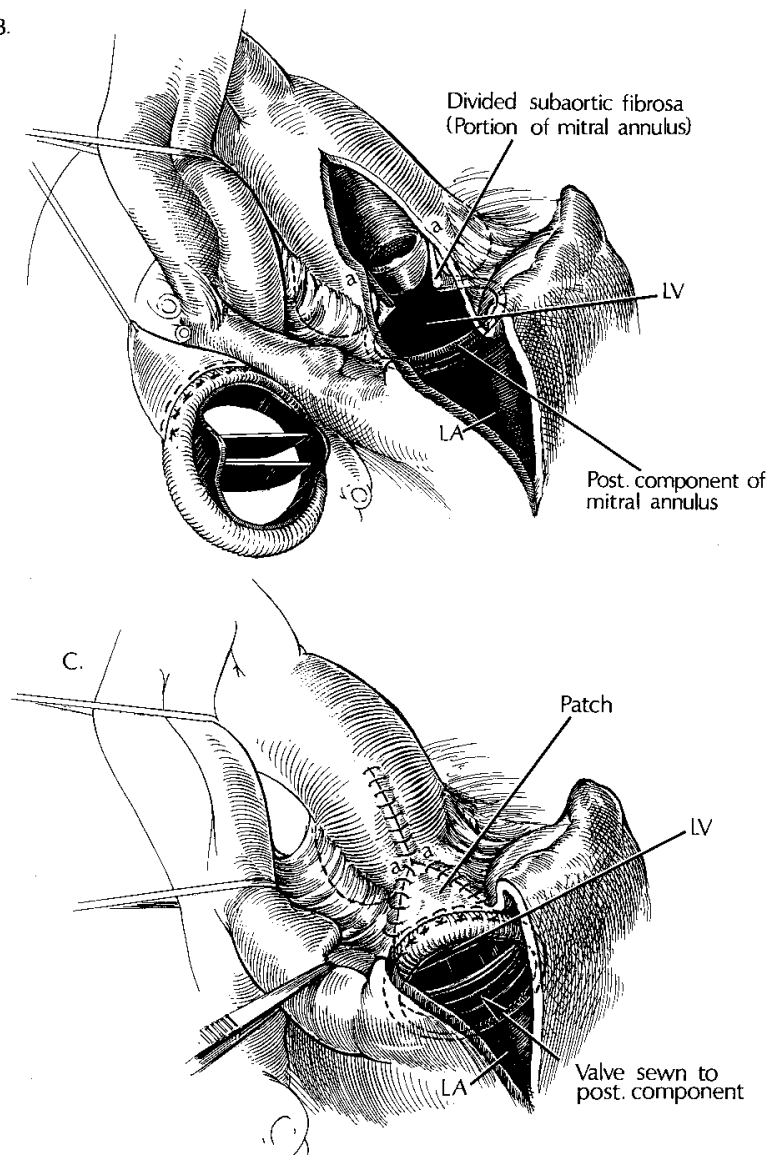

Fig. 1A. View of the heart from the right. An incision in the right lateral aspect of the aorta is carried through the commissure between the left and noncoronary cusps of the aortic valve. $R A$, Right atrium.

Fig. 1B. Incision in the roof of left atrium $(L A)$ and atrial septum exposes the mitral anulus. The mitral valve has been excised. Points " $a$ " correspond to the tops of the commissure of the left and noncoronary cusps of aortic valve. $L V$, Left ventricle. sis, an alternative approach can be used, as we describe in this report.

Method. Moderately hypothermic bypass is established with the arterial cannula in the ascending aorta and venous return through a right-angled cannula in the superior vena cava and a straight cannula in the inferior vena cava. After the aorta has been crossclamped, cardioplegic solution infused, and caval tourniquets tightened, a vertical incision is made in the right lateral aspect of the ascending aorta (Fig. 1A). The incision is directed toward the commissure between the left and noncoronary cusps. An oblique incision is made in the right atrial free wall and is extended across the roof of the right atrium, across the atrial septum, and into the left atrium so as to meet the aortic incision. The atrial septal incision is extended inferiorly to the inferior margin of the left atrium. The stenotic and hypoplastic mitral valve is excised. The aortic incision is carefully extended across the non-left commissure and completely through the subaortic fibrosa (Fig. 1B). The subaortic area is now completely open for inspection. Any fibrous membranelike tissue can be excised with particular care in the region of the membranous septum to avoid injury to the conduction bundle. A triangular patch of polytetrafluoroethylene is sutured, as shown in Fig. 1C, to reconstitute the aortic valve and to supplement the mitral anulus. The apex of the triangle is at the commissure of the aortic valve, and the broad base of the patch will form part of the mitral anulus. Autologous pericardium treated with glutaraldehyde is useful to extend the aortic valve cusps and to increase the area of aortic leaflet coaptation. A mitral prosthesis is sutured to the base of the prosthetic patch, as well as to the remainder of the mitral anulus. The length of the base of the prosthetic patch determines the degree to which the mitral anulus is enlarged. The atrial septum and the roof of the left atrium are closed with a polytetrafluoroethylene patch. The right atriotomy and aortotomy are closed, supplemented with patches if necessary. The heart is deaired in the usual fashion.

\section{Clinical summaries}

PATIENT 1. A 15-year-old girl had been noted at 7 years of age to have a primum atrial septal defect with severe subaortic stenosis. However, she was not referred for surgery until 9 years of age. At that time the mitral valve, which was the primary cause of the outflow tract obstruction, was resected and replaced with a $23 \mathrm{~mm}$ St. Jude Medical prosthesis (St. Jude Medical, Inc., St. Paul,

Fig. 1C. Triangular prosthetic patch enlarges the mitralanulus and subaortic area. The mitral valve has been replaced with a mitral prosthesis. The native valve is reconstituted at the apex of the patch $(a: a)$. $L A$, Left atrium; $L V$, left ventricle. 
Minn.). The primum atrial septal defect was closed with a pericardial patch. Postoperatively, complete heart block developed and necessitated pacemaker implantation. At the time of pacemaker generator replacement, transesophageal echocardiography revealed a $100 \mathrm{~mm} \mathrm{Hg}$ gradient across the left ventricular outflow tract and mild aortic regurgitation. Cardiac catheterization showed a $65 \mathrm{~mm} \mathrm{Hg}$ gradient, which appeared to be due to a tunnel extending from the aortic valve to the level of the mitral prosthesis. A 4 to $6 \mathrm{~mm} \mathrm{Hg}$ mean gradient was detected across the mitral prosthesis. At operation the aortic valve was noted to be tricuspid, although the left cusp was half the size of the noncoronary cusp. The technique described herein was applied. The mitral valve was again replaced with a $25 \mathrm{~mm}$ St. Jude Medical prosthesis. A collagen-impregnated Dacron patch was used to enlarge the subaortic area and mitral anulus. The non-left aortic commissure of the aortic valve was reconstituted. In this first patient, autologous pericardial leaflet extenders were not used in the aortic valve reconstruction. The child tolerated the procedure well and required no inotropic support. Intraoperative transesophageal echocardiography revealed a residual Doppler gradient of 25 $\mathrm{mm} \mathrm{Hg}$ across the left ventricular outflow tract, no change in the mild aortic regurgitation present before the operation, and a mean gradient of $5 \mathrm{~mm} \mathrm{Hg}$ across the new mitral prosthesis. Both leaflets of the prosthesis moved freely.

At most recent follow-up 4 years after the operation, a $25 \mathrm{~mm} \mathrm{Hg}$ gradient across the left ventricular outflow tract and moderate aortic regurgitation were detected by continuous-wave Doppler echocardiography. Inflow velocities were not suggestive of significant stenosis of the mitral prosthesis. Symptomatically, she is well and has recently begun attending college.

PATIENT 2. A 12-year-old boy had been noted in the neonatal period to be in congestive failure. Cardiac catheterization revealed mitral stenosis resulting from a mitral arcade. When the boy was 7 weeks of age the mitral valve was replaced with a $17 \mathrm{~mm}$ Björk-Shiley prosthesis (Shiley, Inc., Irvine, Calif.). At 14 months the prosthesis was replaced with a $17 \mathrm{~mm}$ prosthesis because of pannus ingrowth. When the boy was 6 years of age a third prosthetic valve replacement was performed with a $19 \mathrm{~mm}$ St. Jude Medical prosthesis. A progressive left ventricular outflow gradient was noted to develop after the operation. At catheterization when the boy was 11 years old, there was a $40 \mathrm{~mm} \mathrm{Hg}$ gradient across the left ventricular outflow tract and a $10 \mathrm{~mm} \mathrm{Hg}$ mean gradient across the mitral prosthesis. At operation the aortic valve was noted to be tricuspid, with some thickening of the leaflets but no commissural fusion. The subaortic outflow area was tunnel-like. Left ventricular outflow obstruction was exacerbated by projection of the mitral prosthesis into the outflow tract.

By means of the technique described herein, the subaortic area was laid open. The mitral prosthesis was excised and was replaced with a $21 \mathrm{~mm}$ St. Jude Medical prosthesis. After reconstitution of the aortic valve at the left-noncommissure, a leaflet extender of autolo- gous pericardium treated with glutaraldehyde was sutured to the free edge of the noncoronary cusp to improve leaflet apposition. The child tolerated the procedure well and required no inotropic support. By simultaneous pressure catheter measurement there was a $10 \mathrm{~mm} \mathrm{Hg}$ residual outflow tract gradient and by echocardiography trivial to mild aortic regurgitation. No gradient could be measured across the mitral prosthesis.

At the most recent follow-up catheterization 2 years after the operation, there was a 25 to $30 \mathrm{~mm} \mathrm{Hg}$ gradient across the left ventricular outflow tract and mild to moderate aortic valve regurgitation. The mitral valve area was calculated at $1.3 \mathrm{~cm}^{2} / \mathrm{m}^{2}$. Pulmonary artery pressure was one third systemic pressure. He is free of symptoms and in sinus rhythm.

Discussion. Although several procedures have been described for enlargement of the aortic anulus, such as the Nicks, Manougian, and Konno procedures, ${ }^{2}$ there are very few options for enlargement of the mitral anulus. This is because of the presence of the circumflex coronary artery, membranous ventricular septum, and conduction bundle and the aortic valve, which encircle the mitral anulus. In the past we have performed supraannular mitral valve replacement when the mitral anulus has been inadequate to accept an adequate-sized mitral prosthesis. Supraannular mitral valve replacement involves insertion of the prosthesis entirely within the left atrium, thereby creating a ventricularized portion of the left atrium. Although the early results of this procedure were encouraging, the late survival and late hemodynamic results have been disappointing. ${ }^{3}$ The technique described in this report provides an alternative to supraannular mitral valve replacement and is particularly appropriate in the setting of tunnel-like subaortic stenosis.

Rastan $^{4}$ and others have described a procedure in which both the aortic and mitral anuli are enlarged with a single patch, but this procedure necessitates replacement of both valves. In growing children, we believe strongly in the principle of minimizing the number of prostheses inserted. Therefore, even in a child who has previously had a mitral valve replacement, we believe it is a worthwhile goal to preserve the native aortic valve.

The modified Konno procedure has demonstrated the feasibility of enlarging the immediate subaortic region without the need for replacement of the aortic valve. We have applied the same principle in the technique described in this report. The patch that enlarges the mitral anulus is brought into the commissure between the left and noncoronary cusps of the aortic valve, thereby also enlarging the subaortic area. Although this procedure has the risk of causing some degree of aortic regurgitation and the ultimate need for aortic valve replacement, nevertheless in a growing child it is useful to defer valve replacement. A tolerable degree of aortic regurgitation will serve to accelerate the growth of the aortic anulus. Aortic valvuloplasty techniques such as the use of leaflet extenders with autologous pericardium can limit the degree of aortic regurgitation to a tolerable degree. If an acceptable 
reconstruction of the aortic valve cannot be achieved, placement of a pulmonary autograft in the aortic position sutured to the patch enlarging the mitral anulus would seem to be a reasonable option.

\section{REFERENCES}

1. Kirklin JW, Barratt-Boyes BG. Cardiac surgery, 2nd ed. New York: Churchill Livingstone; 1992. p. 1212.
2. Jonas RA. Radical aortic root enlargement in the infant and child. J Card Surg 1994;9:165-9.

3. Adatia I, Moore PM, Jonas RA, Colan SD, Lock JE, Keane JF. Clinical course and hemodynamic observations after supraannular mitral valve replacement in infants and children. J Am Coll Cardiol 1997;29:1089-94.

4. Rastan D. Aortic and aortic-mitral enlargement. J Thorac Cardiovasc Surg 1995;109:818-9. 